\title{
Histoplasmosis diseminada e infección por VIH: serie de casos en un hospital peruano
}

\author{
Giancarlo Pérez-Lazo, Julio Maquera-Afaray, Christian R. Mejia y Raúl Castillo
}

\section{Disseminated histoplasmosis and HIV infection: Case series in a Peruvian hospital}

Background: Histoplasmosis is an endemic fungal infection in Peru and HIV coinfection leads to difficult diagnoses and high associated mortality. Aim: To describe clinical, epidemiological and clinical outcomes in patients with HIV infection with diagnosis of disseminated histoplasmosis (DH) at Guillermo Almenara Irigoyen National Hospital, Lima, Peru. Methods: Retrospective, descriptive study; information was obtained from the computer registry of patients with HIV infection diagnosed with DH, during the period 1996-2014. Clinical, epidemiological, treatment and outcome characteristics were described. Results: We found 27 patients, $25(92.6 \%)$ were male, with a mean age of 36.7 years $( \pm 9.4$ years $)$ and $22(81.5 \%)$ had an epidemiological history contact. Sixteen patients $(59.3 \%)$ had DH as an AIDS defining disease. The median CD4 count was 65 cells $/ \mathrm{mm}^{3}$ (IQR $\left.15-92\right)$. Only $7(25.9 \%)$ received combination antiretroviral therapy at diagnosis. The most common clinical presentation was fever $(66.7 \%)$, chronic diarrhea (40.7\%) and lymphadenopathy $(33.3 \%)$. The diagnosis was made mainly by histopathology. Six $(22.2 \%)$ patiens died in the acute stage of the disease. Seven $(25.9 \%)$ were initially treated empirically as tuberculosis. Conclusion: We describe the clinical characteristics of a group of patients who had $\mathrm{DH}$ as HIV coinfection and this is still a quasi endemic reality in patients suffering from this pathology.

Key words: Histoplasmosis, AIDS, HIV infection; Peru (source MeSH NLM).

Palabras clave: Histoplasmosis, VIH/SIDA; Perú (fuente: DeCS BIREME).

\section{Introducción}

\section{$\mathrm{L}$} a histoplasmosis es una infección fúngica endémica y oportunista descrita en pacientes con infección por VIH, y aún durante la era post-terapia anti-retroviral combinada (TARc). Tanto la morbilidad como la mortalidad continúan siendo un problema de salud pública en ciertas áreas consideradas endémicas de Sudamérica ${ }^{1-3}$, alcanzando una mortalidad entre 19 y $39 \%$, principalmente en la forma clínica de histoplasmosis diseminada (HD) ${ }^{4,5}$. Asimismo, el problema engloba la dificultad para la sospecha clínica, tratamientos previos empíricos como tuberculosis (TBC), diagnóstico tardío por la falta de laboratorios implementados, diagnóstico tardío de la infección por VIH, retraso en el inicio del tratamiento antifúngico y no disponibilidad de formulaciones lipídicas de anfotericina $\mathrm{B}^{1,3-6}$.

Actualmente es considerada una enfermedad desatendida en Sudamérica ${ }^{1,4,6}$, y la mayoría de las series reportadas provienen de Brasil, Guayana Francesa, Colombia y Argentina ${ }^{7-11}$. En Perú no existen estudios ni series de casos que evalúen el comportamiento de la co-infección HD y VIH. Por tanto, el objetivo del presente estudio fue describir las características epidemiológicas, clínicas, diagnósticas y terapéuticas, así como el desenlace clínico en pacientes con infección por VIH con diagnóstico de HD en un hospital de referencia nacional de la seguridad social en Lima, Perú.

\section{Métodos}

Se analizaron en forma retrospectiva los registros electrónicos de pacientes con infección por VIH con diagnóstico de HD durante el período 1996-2014. El Hospital Nacional Guillermo Almenara Irigoyen (HNGAI) de Lima, Perú pertenece a la seguridad social y es centro de referencia nacional para atención de pacientes con infección por VIH, no siendo Lima un área endémica de histoplasmosis. Se incluyó al total de pacientes atendidos en la institución y no se realizaron exclusiones, ya que todos los pacientes tenían la información mínima para que pudiesen ingresar al estudio.

Luego de la elaboración del proyecto, se procedió a revisar las historias clínicas de los pacientes donde se consideraron las variables: edad del paciente (en años cumplidos), sexo, antecedente epidemiológico, recuento de LT CD4+, uso de TARc previa al diagnóstico, método diagnóstico, características clínicas y de laboratorio, tiempo de demora diagnóstica, tratamiento y desenlace clínico (mortalidad).

Estos datos fueron llevados a una base, que fue generada en el programa Microsoft Excel (para versión Windows 2013). Luego se pasó la información al programa Stata versión 11,1 (StataCorp LP, College Station, TX, USA). Para la estadística descriptiva se usaron frecuencias y porcentajes para las variables categóricas, y la media y desviación estándar para las variables cuantitativas; en
Hospital Guillermo Almenara, Lima, Perú.

Servicio de Medicina InternaInfectología (GPL, JMA, RC). Universidad Privada de Tacna, Tacna, Perú.

Escuela de Medicina (JMA). Universidad Continental, Huancayo, Perú.

Escuela de Medicina (RC).

Los autores declaran no tener conflictos de interés con la publicación de este artículo.

Fuente de financiamiento: Autofinanciado.

Recibido: 14 de febrero de 2017 Aceptado: 10 de julio de 2017

Correspondencia a: Giancarlo Pérez Lazo giancarlo.perez@unmsm.edu.pe diamantdust@hotmail.com 
algunos casos se obtuvo el rango intercuartílico (RIQ). Se pasaron todas éstas a tablas y figuras. El proyecto de investigación fue enviado y aprobado en su revisión por un Comité de Ética local, avalado por el Instituto Nacional de Salud del Perú.

Tabla 1. Características clínicas, epidemiológicas y de laboratorio en 27 pacientes con infección por VIH e HD en el HNGAI 1996-2014

\begin{tabular}{|c|c|}
\hline & Valores \\
\hline \multicolumn{2}{|l|}{ Características demográficas y de VIH en la admisión } \\
\hline Sexo masculino n (\%) & $25(92,6)$ \\
\hline Edad media $\pm D E$, años & $36,7( \pm 9,4)$ \\
\hline Histoplasmosis como enfermedad indicadora de SIDA, $n(\%)$ & $16(59,26)$ \\
\hline Paciente con TARc, n (\%) & $7(25,93)$ \\
\hline Media LT CD4+ (RIQ 25 -75) / $/ \mathrm{mm}^{3}$ & $65(15-92)^{*}$ \\
\hline Antecedente epidemiológico n (\%) & $22(81,48)$ \\
\hline \multicolumn{2}{|l|}{ Síntomas y signos $\mathrm{n}(\%)$} \\
\hline Fiebre & $18(66,7)$ \\
\hline Diarrea crónica & $11(40,7)$ \\
\hline Adenomegalias generalizadas & $9(33,3)$ \\
\hline Disnea & $8(29,6)$ \\
\hline Pérdida de peso & $8(29,6)$ \\
\hline Hepato-esplenomegalia & $5(18,5)$ \\
\hline Tos & $5(18,5)$ \\
\hline Lesiones mucosas & $4(14,8)$ \\
\hline Lesiones en piel & $3(11,1)$ \\
\hline Sangrado digestivo & $3(11,1)$ \\
\hline \multicolumn{2}{|l|}{ Resultados de laboratorio } \\
\hline Hemoglobina, $g / d L,(n=19)$, promedio $\pm D E$ & $8,28( \pm 2,48)$ \\
\hline Plaquetas $/ \mathrm{mm}^{3}(\mathrm{n}=18)$, mediana $(\mathrm{RIQ})$ & $95.000(65.000-135.000)$ \\
\hline Casos confirmados de co-infección TBC/HD n (\%) & $3(11,1)$ \\
\hline Tiempo de demora diagnóstica (días/RIQ) & $30(18-36)$ \\
\hline
\end{tabular}

*No se encontró el recuento de LT CD4+ inicial de dos pacientes. DE: Derivación estándar; TARc: Terapia antiretroviral combinada; RIQ: Rango intercuartílico; TBC: tuberculosis; HD: histoplasmosis diseminada; HNGAl: Hospital Nacional Guillermo Almenara Irigoyen.

Tabla 2. Diagnóstico y localización anatómica de histopatología en 27 pacientes con infección por VIH e HD en el HNGAI 1996-2014

\begin{tabular}{lr}
\hline $\begin{array}{l}\text { Métodos diagnósticos de infección por histoplasma } \\
\text { Cultivo del hongo, n (\%) }\end{array}$ & Valores \\
Detección de antígeno histoplasma (orina), n (\%) & $2(7,41)$ \\
Patología (tinción PAS), n (\%) & $23(85,41)$ \\
& \\
Localización anatómica de la muestra1 n/N (\%) & \\
Mucosa [colon (6), recto (2), paladar (2)] & $10 / 25(40)$ \\
Médula ósea & $4 / 25(16)$ \\
Ganglio [cervical (2), inguinal (1), retroperitoneal (1)] & $4 / 25(16)$ \\
Pulmón [bronquio (1), LBA (2)] & $3 / 25(12)$ \\
Otros [piel (3), cerebro (1)] & $4 / 25(16)$ \\
\hline
\end{tabular}

'No se consideraron dos pacientes pues el diagnóstico sólo fue por antígeno de histoplasma en orina. PAS: ácido peryódico de Schiff; BAL: lavado bronquio-alveolar; HNGAl: Hospital Nacional Guillermo Almenara Irigoyen.

\section{Resultados}

En el período de estudio se encontraron 27 pacientes con HD, 25 (92,6\%) fueron varones, con edad media de 36,7 años $( \pm 9,4$ años) y $22(81,5 \%)$ tuvieron antecedentes epidemiológicos. El 48,1\% $(n=13)$ eran procedentes de zonas endémicas (de las ciudades de Huánuco, Pucallpa e Iquitos) y $33,3 \%(\mathrm{n}=9)$ referían historia de viaje a selva central (región endémica en el Perú). En la mayoría de éstos últimos se recuperó el dato de visita a la "Cueva de las Lechuzas" (gruta que alberga diversas especies silvestres en el Parque Nacional de Tingo María). Sólo uno manifestó como ocupación de riesgo, el ser leñador. El promedio de LT CD4+ fue de 65 céls/mm³ (RIQ: 15-92) y 16 (59,3\%) tuvieron HD como enfermedad indicadora de SIDA.

Los síntomas predominantes fueron fiebre $66,7 \%$ (n: 18 ), diarrea crónica $40,7 \%$ (n: 11) y adenopatías generalizadas 33,3\% (n: 9). Las manifestaciones cutáneas se presentaron en $25 \%$ (n: 7 ) de los casos y el sangrado digestivo bajo en 11,1\% (n: 3). Adicionalmente se encontró pancitopenia en $44.4 \%$ (n: 12 ) de los casos, $66,7 \%$ $(12 / 18)$ cursaron con plaquetas $\leq 100,000 / \mathrm{mm}^{3}$ y $31,5 \%$ (6/19) tuvieron hemoglobina $\leq 8 \mathrm{mg} / \mathrm{dl}$. Las características clínicas y de laboratorio se muestran en la Tabla 1.

El 85,2\% (n: 23) de los pacientes presentó enfermedades oportunistas concomitantes, $18,5 \%$ (n: 5) tuvo herpes simplex diseminado, 11,1\% (n: 3) con TBC pulmonar y extrapulmonar, 11,1\% (n: 3) neumonía por Pneumocystis jiroveci, y la misma cantidad tuvo candidiasis orofaríngea y/o esofágica. Es importante considerar que 25,9\% (n: 7) de los pacientes recibieron tratamiento empírico inicial para TBC y sólo se confirmaron tres casos durante la hospitalización. Además, sólo siete pacientes recibían TARc al momento de la hospitalización (la mayoría de ellos con adherencia irregular), y tres pacientes presentaron síndrome inflamatorio de reconstitución inmune (SIRI) asociado a histoplasmosis.

La localización anatómica más frecuente para obtener las muestras fue la mucosa gastrointestinal con $40 \%$ (n: 10), seguidos de médula ósea y ganglio en $16 \%$ (n: 4), cada una (Tabla 2). El tiempo de demora diagnóstica promedio fue de 30 días (RIQ: 18-36). El principal método diagnóstico fue la histopatología, usando la tinción PAS (ácido peryódico de Schiff) en 85,2\% (n: 23) de los casos. Se realizó cultivo en dos muestras, de las cuales sólo una fue positiva. En los dos casos restantes, el diagnóstico se realizó mediante antígeno para histoplasma en orina, el que fue solicitado en un laboratorio de referencia fuera de la institución. El cultivo positivo se obtuvo de lavado bronquio-alveolar con un tiempo de demora de seis semanas para el reporte, mientras que los resultados de las dos muestras de antígeno para histoplasma fueron reportados a los 10 días.

De los 27 casos estudiados, 88,9\% (n: 24) alcanzó a recibir algún tipo de tratamiento; $62,5 \%$ (n: 15$)$ iniciaron 
con anfotericina $\mathrm{B}$ deoxicolato $0,7-1 \mathrm{mg} / \mathrm{kg} /$ día por una a dos semanas, y 13 continuaron con itraconazol (200 mg VO tres veces/día por tres días, luego $200 \mathrm{mg}$ VO dos veces/día por un año). 33,3\% (n: 8) iniciaron tratamiento con itraconazol con las dosis descritas previamente (iniciado en formas no graves o por no disponibilidad de anfotericina) y sólo un paciente recibió anfotericina B complejo lipídico $5 \mathrm{mg} / \mathrm{kg} /$ día por dos semanas, continuando con el esquema de itraconazol. El 53,3\% (n: 8) de los que recibieron anfotericina $\mathrm{B}$ deoxicolato presentaron toxicidad asociada (por sobre todo hipokalemia y elevación de la creatinina). Tres pacientes no recibieron tratamiento antifúngico pues el diagnóstico de HD fue realizado por necropsia.

Del total, 22,2\% (n: 6) fallecieron en la etapa aguda de la enfermedad (período de los 30 primeros días de iniciada la terapia antifúngica), la que estuvo relacionado con síndrome de distrés respiratorio en tres casos (co-infección con Pneumocystis jiroveci) y hemorragia digestiva baja con trastorno de la coagulación en tres casos. Luego del primer mes de tratamiento antifúngico fallecieron dos pacientes por complicaciones secundarias a TBC extrapulmonar (meningitis con hidrocefalia más neumonía aspirativa y hemorragia cerebral, respectivamente), sin mención de inicio de TARc.

Fueron dados de alta 16 pacientes, de los cuales seis efectuaron controles posteriores en nuestro nosocomio, ocho fueron referidos para completar tratamiento con itraconazol en otro hospital de referencia y dos no efectuaron controles posteriores al egreso hospitalario.

El inicio de TARc en 10 de ellos se realizó luego de las dos semanas de tratamiento antifúngico, y en tres pacientes luego de 30 días de iniciado el tratamiento. Los tres pacientes que presentaron SIRI continuaron con el mismo esquema. Seis pacientes en seguimiento en nuestra institución completaron tratamiento con itraconazol por un año, con carga viral indetectable para VIH.

\section{Discusión}

La HD constituye la forma clínica más frecuente de histoplasmosis en pacientes con infección por VIH y está asociada a recuentos bajos de $\mathrm{LT} \mathrm{CD} 4+3^{3}$, como ocurre en países de Latinoamérica y el Caribe, incluyendo Perú, donde existe una alta tasa de inicio tardío de la TARc ${ }^{12,13}$. Estudios previos en la región fueron reportados en Brasil, Guayana Francesa y Panamá registrando 164, 82 y 104 casos de HD, respectivamente ${ }^{7,9,14}$. Esta serie de casos de HD en pacientes con infección por VIH es la primera en describir las características epidemiológicas y clínicas de esta enfermedad en Perú.

Casi más de la mitad de los casos de HD en nuestra serie se presentaron como enfermedad indicadora de SIDA, y la mayoría no se encontraba recibiendo TARc al momento del diagnóstico; asimismo, afectó más a varones jóvenes y con inmunosupresión grave, coincidente con otras series latinoamericanas ${ }^{4,8,9,11,14}$. Poco más de $80 \%$ manifestó exposición de riesgo epidemiológico con áreas endémicas descritas en Perú; como lo son Huánuco, Pucallpa e Iquitos ${ }^{15}$, siendo la visita al atractivo turístico principal del Parque Nacional de Tingo María conocido como la "cueva de las lechuzas", la exposición de riesgo peruana clásicamente reconocida para histoplasmosis ${ }^{16}$. Además, se han descrito exposiciones de riesgo ocupacional como la minería, tala de árboles, exploración espeleológica, entre otros; sin embargo, encontramos que sólo un paciente presentó ocupación de riesgo, similar a lo descrito por Velásquez y cols. ${ }^{11}$, lo cual no debe limitar la sospecha clínica de esta enfermedad.

El cuadro clínico principal estuvo caracterizado por fiebre, adenomegalias y diarrea crónica, semejante con la serie descrita por Putot y cols. ${ }^{9}$; no obstante, la presencia de síntomas respiratorios como disnea y tos, así como visceromegalia y falla renal fueron más frecuentes en otros estudios latinoamericanos ${ }^{8,11,13}$. La identificación de la pancitopenia podría ser una característica importante para el planteamiento diagnóstico de HD en pacientes con infección por VIH, fundamentalmente plaquetopenia, descrita incluso por otros estudios como factor de riesgo asociado a mortalidad ${ }^{17}$.

En Perú se han reportado cuadros de hemorragia digestiva baja, debido a lesiones ulcerativas colónicas por Histoplasma capsulatum, como una forma infrecuente de presentación; cabe mencionar que el compromiso colónico fue el más frecuente en nuestra serie, y recopilamos tres eventos similares de hemorragia digestiva con desenlace fata $l^{18}$. Por otro lado, el compromiso cutáneo encontrado fue similar a lo descrito en pacientes con infección por VIH $(10-25 \%)^{19}$, con un espectro diverso de lesiones; sin embargo, otras series en Sudamérica reportan frecuencias de hasta $75 \%{ }^{10}$.

Consideramos la demora diagnóstica un serio problema en nuestra serie, con un promedio de 30 días, que tiene implicancias diagnósticas, terapéuticas y pronósticas. En la mayoría de los casos descritos, el diagnóstico de HD se hizo por histopatología (tinción PAS) y sólo un paciente presentó cultivo positivo, siendo esta última una prueba de referencia diagnóstica con una sensibilidad cercana a $75 \%$, en el caso de formas diseminadas ${ }^{11,20,21}$. No obstante, estos métodos diagnósticos -especialmente la histopatología-suelen demorar e incluso brindar características semejantes a otras enfermedades granulomatosas (como la TBC y otras micosis endémicas), repercutiendo negativamente sobre el tiempo diagnóstico e inicio de una terapia dirigida.

Resaltamos que el estudio de médula ósea nos permitió el diagnóstico oportuno en la quinta parte de los casos, corroborando su utilidad para la identificación temprana de histoplasmosis ${ }^{22}$. Está descrito que el mayor rendimiento para las muestras de patología se consigue de muestras respiratorias y de biopsia de médula ósea, 
sobre todo en pacientes con HD, con sensibilidad descrita hasta en $76 \%{ }^{20,23}$. Igualmente, los cultivos son más útiles en pacientes con HD o histoplasmosis pulmonar crónica, siendo positivos en 50 a $85 \%$ de los casos. El mayor rendimiento para cultivo es de muestras de sangre o médula ósea, siendo positivos en $75 \%$ de los $\operatorname{casos}^{1,20,23}$.

Existen pruebas diagnósticas con mayor rentabilidad como es la detección del antígeno de Histoplasma en orina, examen no disponible en instituciones públicas en nuestro país, pero que debe considerarse como una herramienta útil. Esta prueba es un enzimoinmunoensayo basado en la detección del antígeno polisacárido de $H$. capsulatum en materiales biológicos como orina, suero, y fluido de lavado bronquio alveolar. Existen dos pruebas MVista (no disponible comercialmente) en Indianapolis, e IMMY (disponible comercialmente desde 2006) en Norman. Además, en un reciente meta-análisis, determinaron que tanto la antigenuria como la antigenemia tienen un valor diagnóstico similar en histoplasmosis. La sensibilidad para antigenuria y antigenemia fue de 79\% (95\% IC 76-82\%) y $82 \%$ (95\% IC 79-85\%), respectivamente. Los valores de especificidad fueron $99 \%$ (95\% IC 98-100\%) en orina y 97\% (95\% CI 96-98\%) en suero ${ }^{24}$. El fabricante recomienda que esta prueba no debe ser la única herramienta diagnóstica pues hay reacción cruzada con blastomicosis, paracoccidioidomicosis, peniciliosis, esporotricosis, coccidioidomicosis (menos frecuente) y aspergilosis (raro) ${ }^{25}$. También, puede ser usado para monitorizar la respuesta al tratamiento antifúngico ${ }^{1}$.

La existencia de co-infección TBC/histoplasmosis se estima que ocurre en 8 a $15 \%$ de las personas infectadas con $\mathrm{VIH}^{5,26}$. De manera similar, encontramos $11 \%$ de esta co-infección; no obstante, resulta preocupante que adicionalmente cerca de $30 \%$ de los casos recibieran tratamiento anti-TBC sin tener un diagnóstico microbiológico definido. Ciertamente, la $\mathrm{TBC}$ es aún un importante problema para la salud global, y en la Región de Las Américas y el Caribe, Perú se encuentra entre los países con más altas tasas de incidencia de TBC sensible y multi-drogo-resistente ${ }^{27}$. Además, tras el advenimiento del VIH, la co-infección $\mathrm{TBC} / \mathrm{VIH}$ ha incrementado significativamente el riesgo de morbilidad y mortalidad, siendo incluso la principal causa de admisión hospitalaria en personas con infección por $\mathrm{VIH}^{28}$. Es importante recordar que ambas enfermedades oportunistas pueden producir un cuadro clínico semejante, dificultando y retrasando el diagnóstico de histoplasmosis ${ }^{26,29}$; por tanto, deben excluirse apropiadamente diagnósticos alternativos, más aún en países con alta incidencia de TBC, existencia de riesgo epidemiológico de histoplasmosis y donde el diagnóstico no ha sido definido. Asimismo, ambas enfermedades pueden coexistir, y en esta serie encontramos tres pacientes con TBC e HD, dos con desenlace fatal.

El diagnóstico de HD fue post-mortem en tres pacientes, semejante a lo encontrado en un reporte de necropsias en pacientes con infección por VIH realizado en un hospital público peruano ${ }^{30}$, donde más de $50 \%$ de los pacientes tuvieron una enfermedad relacionada con el SIDA, que no había sido sospechada clínicamente pre-mortem.

Más de $60 \%$ de pacientes con HD recibieron anfotericina $\mathrm{B}$, y continúa siendo el régimen inicial de primera opción, como en otras cohortes sudamericanas ${ }^{11}$; las formulaciones lipídicas han demostrado mayor efectividad en pacientes co-infectados ${ }^{20}$. A su vez, el uso previo de TARc es considerado un factor protector encontrado en otros estudios; no obstante, sólo una cuarta parte de nuestros pacientes la recibían ${ }^{17}$.

Sobre la mortalidad de los pacientes co-infectados $\mathrm{VIH} / \mathrm{HD}$, surge el concepto mortalidad temprana definida como aquella ocurrida dentro de los 30 días de iniciado el tratamiento antifúngico, pues es difícil determinar retrospectivamente si la muerte estuvo vinculada directamente a la HD, debido a la presencia de otras enfermedades oportunistas concomitantes y al grado de inmunosupresión grave ${ }^{4}$. Nosotros reportamos que uno de cada cinco pacientes tuvo una muerte en el período agudo de la enfermedad; sin embargo, la HD es una enfermedad crónica con un tratamiento prolongado (por lo menos 1 año); y existe un riesgo de recaída de hasta $23,3 \%$ en series brasileñas ${ }^{8,9}$, siendo la mala adherencia a la TARc un factor claramente asociado, tanto en mortalidad general como en recaída. La mortalidad tardía en este grupo de pacientes aún no está claramente estudiada.

El estudio tuvo la limitación del sesgo de selección; al ser una serie de casos de un determinado hospital no se puede inferir que estas características sean similares a todos los pacientes peruanos que tengan la enfermedad. Sin embargo, al ser extraídos de uno de los hospitales más grandes del Perú y por haber usado un gran rango de período de tiempo, se puede decir que estos resultados podrían estar cercanos a la realidad. Se recomienda que se generen más investigaciones, que puedan incluir a pacientes de otras instituciones importantes del país.

En conclusión, casi la totalidad de pacientes fueron varones, con edad media de 36,7 años y la gran mayoría tuvo antecedente epidemiológico. Seis de cada diez pacientes tuvieron HD como enfermedad indicadora de SIDA. La mediana de LT CD4+ fue de 65 céls $/ \mathrm{mm}^{3}$ y sólo uno de cada cuatro pacientes recibían al momento del diagnóstico TARc. La presentación clínica más frecuente fue la fiebre, seguido de la diarrea crónica y las linfoadenopatías; con diagnóstico realizado principalmente por histopatología. Uno de cada cinco falleció en la etapa aguda de la enfermedad, y un cuarto de la población fue tratada inicialmente de forma empírica como TBC.

\section{Resumen}

Introducción: La histoplasmosis es una infección fúngica endémica en Perú, y la co-infección con VIH conlleva 
dificultades diagnósticas y alta mortalidad asociada. Objetivo: Describir las características clínicas, epidemiológicas y desenlace clínico en pacientes con infección por VIH con diagnóstico de histoplasmosis diseminada (HD) en el Hospital Nacional Guillermo Almenara Irigoyen, Lima-Perú. Material y Métodos: Estudio retrospectivo, descriptivo; se obtuvo información del registro informático de pacientes con infección por VIH con diagnóstico de HD, durante el período 1996-2014. Se describieron características clínicas, epidemiológicas, tratamiento y desenlace. Resultados: Se encontraron 27 pacientes, $25(92,6 \%)$ fueron varones, con edad media de 36,7 años ( $\pm 9,4$ años) y $22(81,5 \%)$ tuvieron antecedente epidemiológico. Dieciséis $(59,3 \%)$ tuvieron
HD como enfermedad indicadora de SIDA. El recuento medio de LT CD4+ fue de 65 céls $/ \mathrm{mm}^{3}$ (RIQ 15-92). Sólo siete $(25,9 \%)$ recibían al momento del diagnóstico terapia anti-retroviral combinada. La presentación clínica más frecuente fue con fiebre $(66,7 \%)$, diarrea crónica $(40,7 \%)$ y linfoadenopatías $(33,3 \%)$; el diagnóstico fue realizado principalmente mediante histopatología. Seis $(22,2 \%)$ fallecieron en la etapa aguda de la enfermedad. Siete $(25,9 \%)$ fueron tratados inicialmente de forma empírica como tuberculosis. Conclusiones: Se describen las características clínicas de un grupo de pacientes que tuvieron HD como co-infección de VIH, siendo esto aún una realidad cuasi endémica en los pacientes que sufren esta patología.

\section{Referencias bibliográficas}

1.- Adenis A, Aznar C, Couppié P. Histoplasmosis in HIV-infected patients: a review of new developments and remaining gaps. Curr Trop Med Rep 2014; 1: 119-28.

2.- Colombo A, Tobón A, Restrepo A, QueirozTelles F, Nucci M. Epidemiology of endemic systemic fungal infections in Latin America. Med Mycol 2011; 49 (8): 785-98.

3.- Gómez B L. Histoplasmosis: epidemiology in Latin America. Curr Fungal Infect Rep 2011; 5: 199-205.

4.- Adenis A, Nacher M, Hanf M, Vantilcke V, Boukhari R, et al. HIV-associated histoplasmosis early mortality and incidence trends: from neglect to priority. PLoS Negl Trop Dis 2014; 8 (8): e3100.

5.- Couppie P, Aznar C, Carme B, Nacher M American histoplasmosis in developing countries with a special focus on patients with HIV: diagnosis, treatment, and prognosis. Curr Opin Infect Dis 2006; 19: 443-9.

6.- Nacher M, Adenis A, Mc Donald S, Do Socorro Mendonca Gomes M, Singh S, et al. Disseminated histoplasmosis in HIV-infected patients in South America: a neglected killer continues on its rampage. PLoS Negl Trop Dis 2013; 7(11): e2319.

7.- De Francesco Daher E, De Sousa Barros F A, Da Silva G B Jr, et al. Risk factors for death in acquired immunodeficiency syndromeassociated disseminated histoplasmosis. Am J Trop Med Hyg 2006; 74: 600-3.

8.- Pontes L B, Leitao T M J S, Lima G G, Gerhard E S, Fernandes T A. Clinical and evolutionary characteristics of 134 patients with disseminated histoplasmosis associated with AIDS in the State of Ceará. Rev Soc Bras Med Trop 2010; 43 (1): 27-31.

9.- Putot A, Perrin S, Jolivet A, Vantilcke V. HIV-associated disseminated histoplasmosis in western French Guiana. 2002-2012. Mycoses 2015; 58: 160-6.

10.- Pietrobon D, Negro-Marquínez L, Kilstein J, Galíndez J, Greca A, Battagliottu C. Histoplasmosis diseminada y SIDA en un hospital argentino: manifestaciones clínicas, diagnóstico y tratamiento. Enferm Infecc Microbiol Clin 2004; 22 (3): 156-9

11.- Velásquez G, Rueda Z V, Vélez L A, Aguirre D A, Gómez-Arias R D. Histoplasmosis en pacientes con SIDA. Un estudio de cohorte en Medellín, Colombia. Infectio; 2010; 14 (S2): S99-S106.

12.- Crabtree-Ramírez B, Caro-Vega Y, Shepherd BE, Wehbe F, Cesar C, Cortes C, et al. Crosssectional analysis of late HAART initiation in Latin America and the Caribbean: late testers and late presenters. PLoS ONE 2011; 6 (5): e20272.

13.- Maquera-Afaray J, Cvetkovic-Vega A, Cárdenas M M, Kälviäinen H, Mejia C R. Diagnóstico tardío y enfermedad avanzada de VIH en pacientes adultos en un hospital de la seguridad social de Perú. Rev Chilena Infectol 2016; 33 (Supl 1): 20-6.

14.- Gutiérrez M E, Canton A, Sosa N, Puga E, Talavera L. Disseminated histoplasmosis in patients with AIDS in Panama: a review of 104 cases. CID 2005; 40: 1199-202.

15.- Inocente A, Aguirre J, Torres R, Sifuentes Y, Salas E. Histoplasmoma ileal en un paciente con Virus de Inmunodeficiencia Humana. Acta Med Peruana 2012; 29 (4): 194-6.

16.- Lazarus A, Ajello, L. Aislamiento de Histoplasma capsulatum del suelo de una cueva en el Perú. Rev. Perú. Med Exp Salud Pública 1955; 9 (1-2): 5-15.

17.- Cáceres D, Gómez B, Restrepo A, Tobón A Histoplasmosis y SIDA: factores de riesgo clínicos y de laboratorio asociados al pronóstico de la enfermedad. Infectio 2012; 16 (3): $44-50$

18.- Alva E, Vásquez J, Frisancho O, Yoza M, Yábar A. Histoplasmosis colónica como manifestación diagnóstica de Sida. Rev Gastroenterol Perú 2010; 30 (2): 163-6.

19.- Peching G, Kumakawa Z, Galarza C. Histoplasmosis diseminada aguda: enfoque de manifestaciones cutáneas. Folia Dermatol Peru 2004; 15 (3): 172-5.

20.- Hage C, Azar M, Bahr N, Loyd J, Wheat J. Histoplasmosis: up-to-date evidence-based approach to diagnosis and management. Semin Respir Crit Care Med 2015; 36: 729-45.
21.- Arango-Bustamante $\mathrm{K}$, Restrepo A, Cano L E, de Bedout C, Tobón A M, González A. Diagnostic value of culture and serological tests in the diagnosis of histoplasmosis in HIV and non-HIV Colombian patients. Am J Trop Med Hyg 2013; 89 (5): 937-42.

22.- Corzo J, Rojas E M, Figueroa C L, Daza N Prevalencia de infecciones oportunistas en pacientes con VIH-SIDA con base en el estudio microscópico de médula ósea. Acta Médica Colombiana 2015; 40 (2): 93-100.

23.- Wheat L J, Azar M M, Bahr N C, Spec A, Relich R F, Hage C. Histoplasmosis. Infect Dis Clin North Am 2016; 30 (1): 207-27.

24.- Fandiño-Devia E, Rodríguez-Echeverri C, Cardona-Arias J, González A. Antigen detection in the diagnosis of histoplasmosis: a meta-analysis of diagnostic performance. Mycopathologia 2016; 181 (3-4): 197-205.

25.- Falci DR, et al. Progressive disseminated histoplasmosis: a systematic review on the performance of nonculture based diagnostic tests. Braz J Infect Dis 2016.

26.- Agudelo C A, Restrepo C A, Molina D A, Tobon A M, Kauffman C A, Murillo C, et al. Tuberculosis and histoplasmosis co-infection in AIDS patients. Am J Trop Med Hyg 2012; 87 (6): 1094-8

27.- Ministerio de Salud del Perú. Análisis de la situación epidemiológica de la tuberculosis en el Perú, 2015. Ministerio de Salud, Dirección General de Epidemiología.2016. http://www. dge.gob.pe/portal/docs/tools/tbc/asistbc.pdf

28.- Ford N, Shubber Z, Meintjes G, Grinsztejn B, Eholie S, Mills E J, et al. Causes of hospital admission among people living with HIV worldwide: a systematic review and metaanalysis. Lancet HIV 2015; 2(10): 438-44.

29.- Qureshi A. A case of histoplasmosis mimicking tuberculosis. J Pakistan Med Assoc 2008; 58 (8): 457-8.

30.- Eza D, Cerrillo G, Castro C, Ticona E, Moraales D, Herrera P M, et al. Resultados post mortem e infecciones oportunistas en pacientes VIH-positivos de un hospital público del Perú. Rev Perú Med Exp Salud Pública 2006; 23 (4): $270-4$ 